Original Research Article

\title{
Evaluation of efficacy and tolerability of nitrofurantoin versus ciprofloxacin in patients of urinary tract infection: a comparative study
}

\author{
Rajneet Kaur', Vijay Kumar ${ }^{1 *}$, Harjinder Singh², Geeta Walia ${ }^{3}$, Arshiya Sehgal ${ }^{4}$
}

${ }^{1}$ Department of Pharmacology, Govt. Medical College Patiala, Punjab, India

${ }^{2}$ Department of Urology, Rajindra Hospital Patiala, Punjab, India

${ }^{3}$ Department of Microbiology, Govt. Medical College Patiala, Punjab, India

${ }^{4}$ Medical Officer, General Hospital Narayangarh, Ambala, Haryana, India

Received: 11 September 2017 Accepted: 03 October 2017

*Correspondence to:

Dr. Vijay Kumar,

Email: vijayksehgal@

yahoo.com

Copyright: (C) the author(s), publisher and licensee Medip Academy. This is an openaccess article distributed under the terms of the Creative Commons Attribution NonCommercial License, which permits unrestricted noncommercial use, distribution, and reproduction in any medium, provided the original work is properly cited.

\section{ABSTRACT}

Background: UTIs are one of the most common infectious diseases encountered in out-patient departments on day to day basis. Nitrofurantoin and Ciprofloxacin are most commonly used antibiotics in the treatment of UTI. The present study was done to compare the efficacy and tolerability of nitrofurantoin and ciprofloxacin in patients of urinary tract infection.

Methods: This prospective, open, randomized, parallel group, comparative study was conducted on 60 patients presenting with acute/uncomplicated or recurrent urinary tract infection in the outpatient Department of Urology, Rajindra Hospital attached to Govt. Medical College, Patiala, Punjab. They were divided into two groups, Group I and Group II of 30 cases each. Group I patients were put on Nitrofurantoin and Group II patients were put on Ciprofloxacin. Initially 100 patients were enrolled but only those who showed growth of uropathogens on baseline urine culture or those who completed the treatment were included in the study. The primary outcome measure was microbiological eradication on post treatment urine culture.

Results: The age range of the patients in Group I and Group II was 19 to 68 years (43.40 \pm 14.58 years) and 20-60 years $(39.77 \pm 13.49$ years) respectively. The total no. of males and females who participated in this study were $32(53.33 \%)$ and 28 $(46.66 \%)$ respectively. The most common uropathogen associated with uncomplicated UTI was E. coli (80\%), other organisms detected were Klebsiella species (16.67\%), Staphylococcus aureus (3.33\%) and Providencia (3.33\%). Post treatment urine culture results showed significant difference between two groups, 5 patients (16.67 \%) in case of Group I and 14 patients (46.67\%) in case of Group II showed growth of micro-organisms post treatment. P-value comes out to be 0.017 which is significant.

Conclusions: In this era of super bugs, nitrofurantoin is more efficacious than ciprofloxacin in the treatment of UTI. E. coli was found to be major organism causing UTI. Ciprofloxacin is less effective due to increasing antibiotic resistance among uropathogens. Both the drugs were well tolerated, no major significant adverse effects were encountered.

Keywords: Ciprofloxacin, Efficacy, Nitrofurantoin, Tolerability, Urinary tract infection

\section{INTRODUCTION}

Uncomplicated urinary tract infections (UTIs) are one of the most frequently encountered infections in out-patient department. Uncomplicated UTIs affect healthy individuals with no other structural/ neurological urinary tract abnormalities. ${ }^{1}$ The most common causative agent of uncomplicated UTI is Escherichia coli. Klebsiella pneumoniae, Enterococcus faecalis, Staphylococcus saprophyticus, group B Streptococcus (GBS), Staphylococcus aureus, Pseudomonas aeruginosa, Proteus mirabilis and Candida are less common causative agents of uncomplicated UTI. 
Lower urinary tract infections present with clinical features such as dysuria, burning during micturition, increased frequency and urgency. ${ }^{2}$ The standard test for diagnosis of urinary tract infection is the detection of uropathogen by urine culture in the presence of clinical symptoms. $^{3}$

Most commonly used antibiotics in the treatment of UTI are trimethoprim- sulfamethoxazole, nitofurantoin, fluoroquinolones, cephalosporins, ampicillin etc. ${ }^{3}$ However, increasing rates of antibiotic resistance and high recurrence rates of these common infections has greatly increased the financial burden of society. ${ }^{1}$

\section{Nitrofurantoin}

Nitrofurantoin (macrocrystals) comes in a larger crystal form. It is currently used to treat uncomplicated UTIs and for prophylaxis against recurrent UTIs. Nitrofurantoin is rapidly reduced to multiple reactive intermediates by flavoproteins (Nitrofuranreductase) inside the bacteria. These reactive intermediates attack ribosomal proteins, DNA, enzymes involved in respiration and pyruvate metabolism within the cell. So, it acts by multiple mechanisms which is likely to be the reason for development of low resistance against this drug. ${ }^{4}$

\section{Ciprofloxacin}

Fluoroquinolones are more commonly prescribed for community-acquired UTIs. Fluoroquinolones are in clinical use for the last two decades. Fluoroquinolones have been considered reasonable empirical agents for both uncomplicated and complicated UTI. ${ }^{5}$ Ciprofloxacin acts by inhibiting bacterial DNA gyrase. ${ }^{6}$ But there are reports of increase in bacterial resistance due to increase in quinolone prescriptions in several different countries. ${ }^{5}$

Keeping in view the mentioned factors, the present study was done to evaluate and compare efficacy and tolerability of Nitrofurantoin versus Ciprofloxacin in patients of urinary tract infection. It is imperative to choose antibiotics wisely because of the increase in antibiotic resistance among micro-organsims.

\section{METHODS}

\section{Study design}

This prospective, open, randomized, parallel group, comparative study was conducted on 60 patients presenting with acute/uncomplicated or recurrent urinary tract infection in the outpatient Department of Urology, Rajindra Hospital attached to Govt. Medical College, Patiala, Punjab. Patients were enrolled in the study after verifying inclusion and exclusion criteria. Initially 100 patients were enrolled but only those who showed growth of uropathogens on baseline urine culture or those who completed the treatment were included in the study. Written informed consent was taken from each
patient.Approval for conducting the study was taken from institutional ethics committee and review board of Govt. Medical College, Patiala, Punjab.

\section{Inclusion criteria}

- Age: 18-70 years

- Gender-male or female

- Patients willing to give written informed consent

- Patients with clinical signs and symptoms of UTI like dysuria, increased frequency, urgency and burning during micturition.

\section{Exclusion criteria}

- Patients presenting with signs and symptoms suggestive of upper urinary tract infection.

- Pregnant females

- History of renal disease

- History of allergy to study drugs

\section{Study sequence}

After taking a thorough history and clinical examination, eligible patients were enrolled in the study. Urine samples were collected and microbiological urine culture was done to detect the growth of any micro-organism. Antibiotic sensitivity testing was also done. Patients showing growth of uropathogen on urine culture were included in study.

Group I patients were put on Nitrofurantoin at a dose of $100 \mathrm{mg}$ BD for 5 days in case of uncomplicated UTI and for 15 days in case of recurrent UTI by oral route and subsequent therapeutic response along with any adverse drug reactions observed in patients were noted. Bacteriological response by urine culture was noted at the end of the study. Group II patients were put on Ciprofloxacin at a dose of $500 \mathrm{mg}$ BD for 5 days in case of uncomplicated UTI and for 15 days in case of recurrent UTI by oral route and subsequent therapeutic response along with any adverse drug reactions observed in patients were noted. Bacteriological response by urine culture was noted at the end of the study.

\section{Urine culture}

Clean-catch, midstream urine samples were collected at the baseline pre-treatment visit and sent to microbiological laboratory. Urine was cultured for growth of any uropathogens by standard loop technique and antibiotic sensitivity testing was done. Treatment was given accordingly. If the urine culture at the post treatment visit showed no growth of uropathogenic organisms, then the treatment was considered to be effective.

\section{Statistical analysis}

The results of observations of individual patients were pooled and analyzed. Statistical analysis was performed using Statistical Program for Social Sciences (SPSS) 
software version 20.0 Chicago, Illinois, USA. For categorical variables chi-square test was used for analysis, p-value was calculated. If it was less than 0.05 , it was considered significant, ' $p$ ' value less than 0.01 was considered highly significant. If it was more than 0.05 , it was considered non-significant.

\section{RESULTS}

The present study was conducted in Rajindra Hospital attached to Govt. Medical College, Patiala with the aim to compare efficacy and safety of Nitrofurantoin and Ciprofloxacin in patients of UTI. The primary outcome measure was microbiological eradication on post treatment urine culture. Initially 100 patients were enrolled but 40 patients dropped out of the study due to various reasons such as no growth of uropathogens at baseline urine culture, didn't take the study medication, lost to follow up or refused to take part in study. So 60 pateints were included in the study and divided into two groups.

The study comprised of Group I and Group II and both groups included 30 patients each.

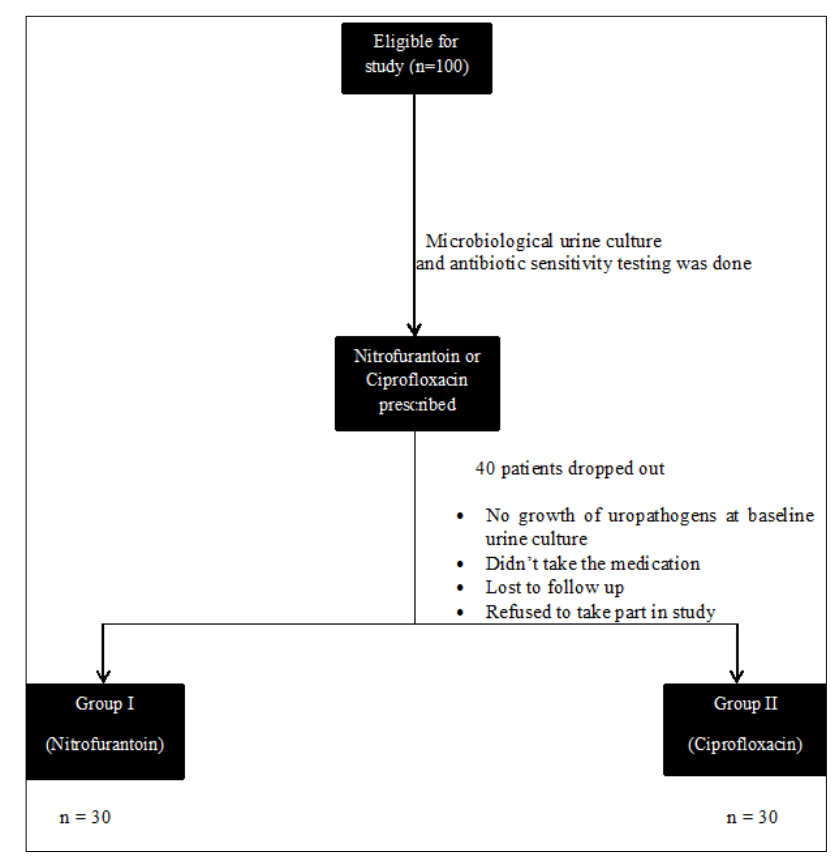

Figure 1: Study sequence.

The age range of the patients in Group I was 19 to 68 years (43.40 \pm 14.58 years) and in Group II was 20-60 years (39.77 \pm 13.49 years). P-value (0.353) for the difference in age range between two groups was not significant.

The total no. of males who participated in this study were $32(53.33 \%)$ and the total number of females were 28 $(46.66 \%)$. Group-wise gender distribution in Group I was: male $(50 \%)$ and female $(50 \%)$ and in Group II was: male $(56.67 \%)$ and female $(43.33 \%)$. P-value $(0.269)$ for the difference in gender distribution between two groups was not significant.

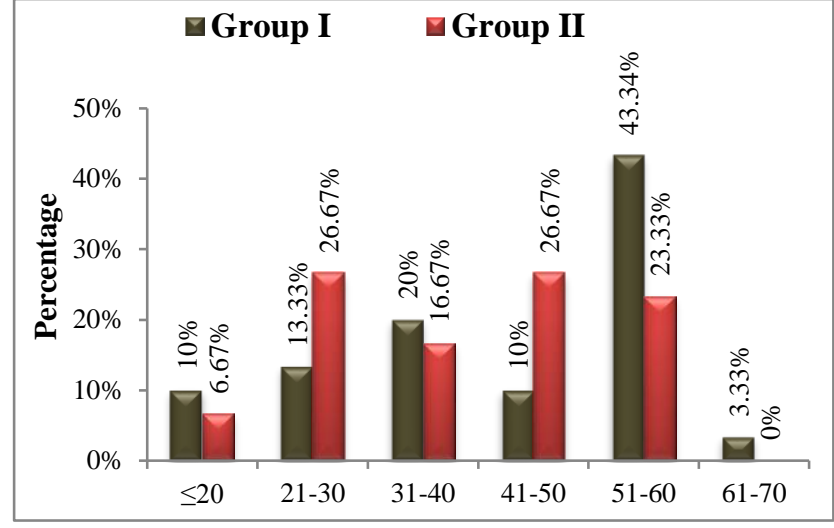

Figure 2: Age distribution in both groups.

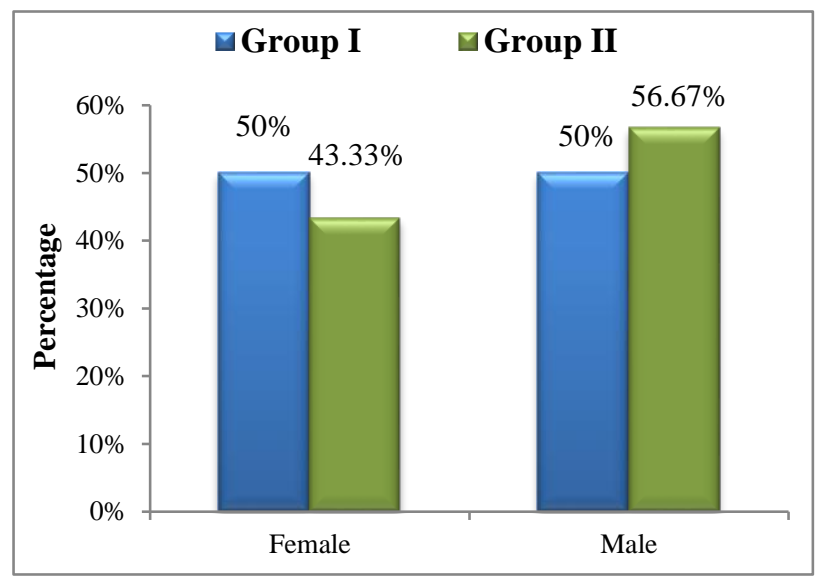

Figure 3: Gender distribution.

The most common uropathogen associated with uncomplicated UTI was E. coli $(80 \%)$, other organisms detected were Klebsiella species (16.67\%), Staphylococcus aureus (3.33\%) and Providencia (3.33\%).

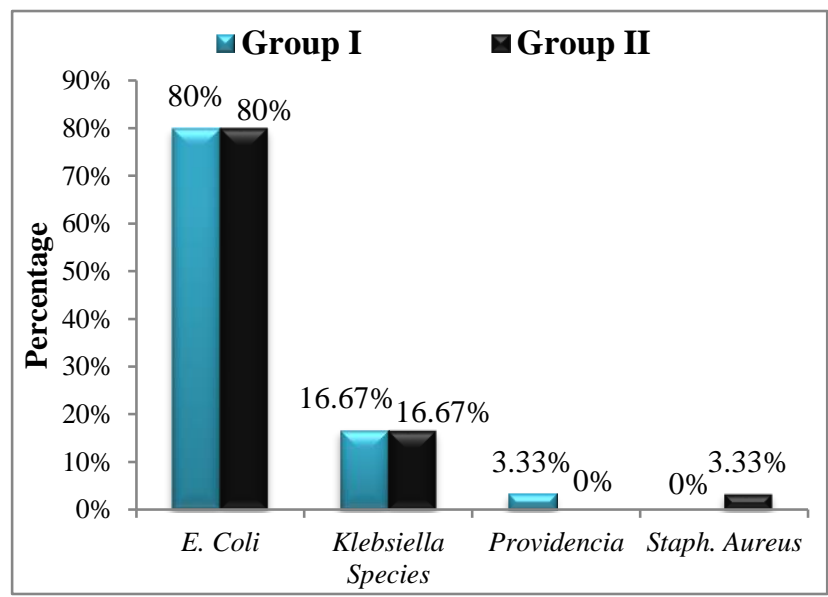

Figure 4: Causative microorganisms for UTI.

Both groups had patients presenting with acute uncomplicated or recurrent UTI, accordingly number of days for which treatment should be given were decided. In Group I, 17 patients $(56.67 \%)$ presented with acute UTI 
and 13 patients $(43.33 \%)$ presented with recurrent UTI. In Group II, 18 patients $(60 \%)$ presented with acute UTI and 12 patients $(40 \%)$ presented with recurrent UTI and accordingly 5 and 15 days of treatment was given respectively. P-value for the difference in no. of days of treatment in both groups is 0.880 , which is not significant.

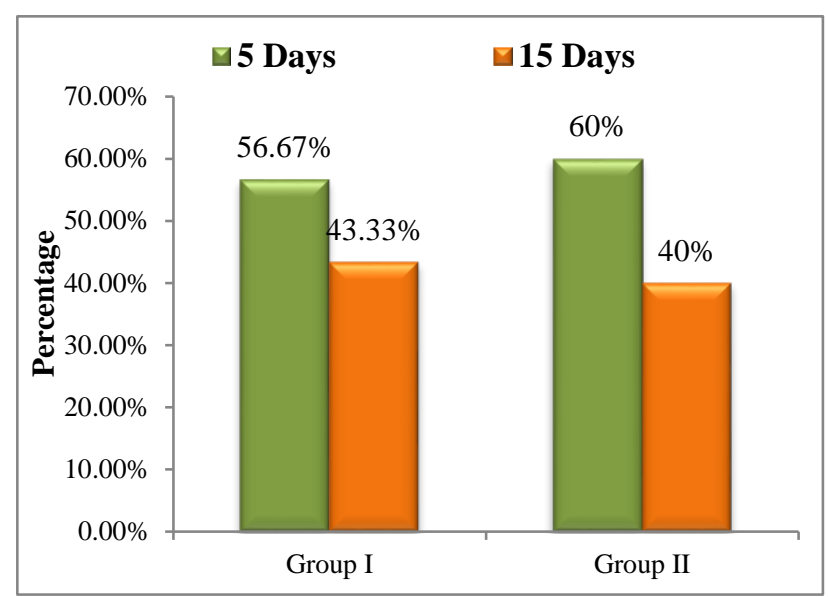

Figure 5: No. of days of treatment for which treatment was given.

Post treatment urine culture results showed significant difference between two groups. If the urine culture was still positive, treatment was considered to be less efficacious. Urine sample of 5 patients $(16.67 \%)$ showed growth post treatment in case of Group I and 14 patients $(46.67 \%)$ in case of Group II showed growth of microorganisms post treatment. P-value comes out to be 0.017 which is significant.

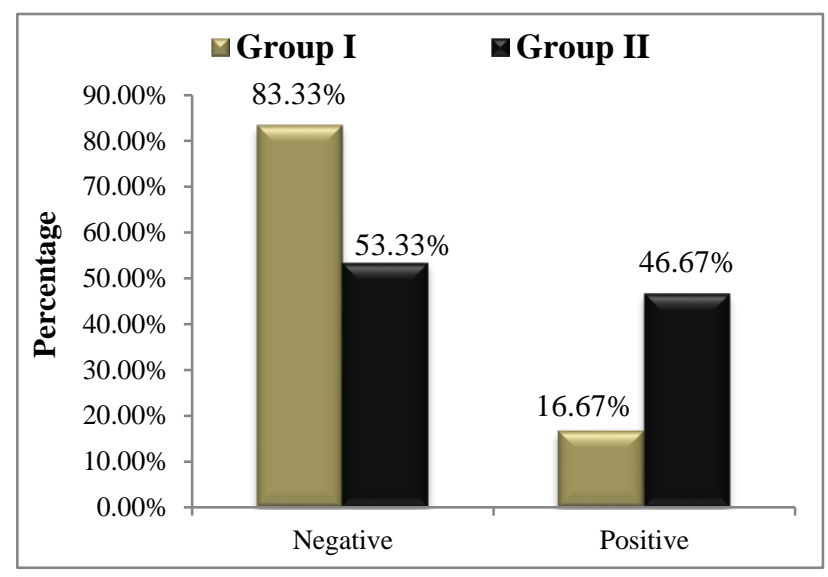

Figure 6: Post-treatment culture results.

Table 1: Safety profile.

\begin{tabular}{|lll|}
\hline Adverse effects & Group I & Group II \\
\hline Nausea or vomiting & $5(16.66 \%)$ & $3(10 \%)$ \\
\hline Headache & $4(13.33 \%)$ & $2(6.66 \%)$ \\
\hline Loss of appetite & $3(10 \%)$ & $2(6.66 \%)$ \\
\hline Abdominal pain & $1(3.33 \%)$ & $1(3.33 \%)$ \\
\hline Diarrhea & $1(3.33 \%)$ & $2(6.66 \%)$ \\
\hline
\end{tabular}

Adverse effects observed in two groups were not of serious nature and there was no significant difference between two groups for the adverse effects reported.

\section{DISCUSSION}

UTIs are common infectious diseases encountered in community practice. Due to increasing rates of antibiotic resistance, there is a need to find an effective treatment for UTI. E. coli is the most common pathogen detected on microbiological urine culture. ${ }^{7}$

In the present study, we have compared efficacy and safety of Nitrofurantoin and Ciprofloxacin. Urine samples were collected from patients presenting with UTI and culturing was done in microbiological lab pre and post treatment. Only positive culture patients were included in the study. The age range of the patients in Group I was 19 to 68 years (43.40 \pm 14.58 years) and in Group II was 20-60 years $(39.77 \pm 13.49$ years). The total no. of males who participated in this study were $32(53.33 \%)$ and the total number of females were $28(46.66 \%)$. Group-wise gender distribution in Group I was: male $(50 \%)$ and female $(50 \%)$ and in Group II was: male (56.67\%) and female (43.33\%). The difference in age and gender distribution between two groups was not significant.

The most common bacteria detected on urine culture was $E$. coli indicating that it is the most common causative agent of urinary tract infections. Treatment was given to both groups. Urine sample of 5 patients $(16.67 \%)$ showed growth post treatment in case of Group I and 14 patients $(46.67 \%)$ in case of Group II showed growth of microorganisms post treatment indicating that nitrofurantoin is more effective in eradicating the bacteria than ciprofloxacin and there are increasing rates of antibiotic resistance against ciprofloxacin.

Safety profile was also compared, few side effects were reported in both the groups and none of them were of the serious nature. This indicates that both drugs are equally tolerable.

In a study conducted by Christiaens, nitrofurantoin was significantly more effective than placebo in achieving bacteriological cure and symptomatic relief in just three days. This is in accordance with our present study. ${ }^{8}$

In a study conducted by Iqbal J, there were increasing rates of antibiotic resistance against ciprofloxacin. Our present study also showed increased resistance against ciprofloxacin. ${ }^{9}$

In a study conducted by Konar $\mathrm{J}$ in 2557 clinically suspected cases, to find out the most common pathogenic bacteria to cause uncomplicated urinary tract infection in a tertiary care hospital. The most common uropathogen to be isolated was E. coli. Majority of the isolated Escherichia coli were sensitive to nitrofurantoin and resistant to Fluoroquinolones. Our present study confirms the same. ${ }^{4}$ 
In a study conducted by Kothari A in India to determine resistance patterns among uropathogens. It showed alarming rate of resistance to antibiotics commonly prescribed for treatment of UTI like Ciprofloxacin, SXT and amoxicillin. ${ }^{10}$

In a study conducted by Spencer RC across the UK in 45 centres including 538 patients, he compared nitrofurantoin MR with trimethoprim or co-trimoxazole and proved nitrofurantoin $\mathrm{MR}$ as a drug of choice for acute and uncomplicated UTI. ${ }^{11}$

In a study conducted by Cagnacci, a total of $148 \mathrm{E}$. coli strains were studied. Most of the bacteria showed resistance to more than two drugs. The drugs most commonly developed resistance was ciprofloxacin, cotrimoxazole, tetracycline and ampicillin. ${ }^{12}$

It can be said that due to increasing antibiotic resistance in E. coli against most commonly used antibiotics, ntirofurantoin is more effective than ciprofloxacin in killing the bacteria. The difference in adverse effect profile was not statistically significant. Therefore, it can be stated that both drugs are equally tolerable.

\section{CONCLUSION}

The results showed that nitrofurantoin is more effective than ciprofloxacin. It causes statistically significant bacteriological improvement on urine culture. Less improvement in case of ciprofloxacin is due to increased antibiotic resistance among uropathogens. Urine sample of 5 patients $(16.67 \%)$ in case of Group I and 14 patients $(46.67 \%)$ in case of Group II showed growth of microorganisms post treatment indicating that nitrofurantoin is more effective. So, antibiotics should be judiciously used, otherwise there would be a lot of financial burden on society to discover new drugs. Both drugs have proven to be equally tolerable.

Funding: No funding sources

Conflict of interest: None declared

Ethical approval: The study was approved by the Institutional Ethics Committee

\section{REFERENCES}

1. Flores-Mireles AL, Walker JN, Caparon M, Hultgren SJ. Urinary tract infections: epidemiology, mechanisms of infection and treatment options. Nat Rev Microbiol. 2015;13(5):269-84.

2. Foxman B. Urinary Tract Infection Syndromes: Occurrence, Recurrence, Bacteriology, Risk Factors, and Disease Burden. Infect Dis Clin N Am. 2014;28:113.
3. Schmiemann G, Kniehl E, Gebhardt K, Matejczyk MM, Hummers-Pradier E. The Diagnosis of Urinary Tract Infection: A Systematic Review. Dtsch Arztebl Int. 2010;107(21):361-7.

4. Konar J, Ghosh R, Chatterjee SS, Majumdar AK, Pathak M, Bhattacharya S. Nitrofurantoin: the timetested choice in uncomplicated urinary tract infection. J Evolution Med Dent Sci. 2016;5(34):1872-5.

5. Arslan H, Azap KO, Ergonul O, Timurkaynak F. Risk factors for ciprofloxacin resistance among Escherichia coli strains isolated from community-acquired urinary tract infections in Turkey. J Antimicrob Chemother. 2005;56:914-18.

6. Fisher LM, Lawrence JM, Josty IC, Hopewell R, Margerrison E, Cullen ME. Ciprofloxacin and the fluoroquinolones: New concepts on the mechanism of action and resistance. Am J Med. 1989;87(5):S2-8.

7. Gupta K, Scholes D, Stamm WE. Increasing prevalence of antimicrobial resistance among uropathogens causing acute uncomplicated cystitis in women. JAMA. 1999;281(8):736-38.

8. Christiaens TCM, Meyere MD, Verschraegen G, Peersman W, Heytens S, Maeseneer JMD. Randomised controlled trial of nitrofurantoin versus placebo in the treatment of uncomplicated urinary tract infection in adult women. $\mathrm{Br} \mathrm{J}$ Gen Pract. 2002;52:729-34.

9. Iqbal J, Rahman M, Kabir MS, Rahmani M. Increasing ciprofloxacin resistance among prevalent urinary tract bacterial isolates in Bangladesh. Jpn J Med Sci Biol. 1997;50:241-50.

10. Kothari A, Sagar V. Antibiotic resistance in pathogens causing community-acquired urinary tract infections in India: a multicenter study. J Infect Developing Countries. 2008;2(5):354-8.

11. Spencer RC, Moseley DJ, Greensmith MJ. Nitrofurantoin modified release versus trimetfaoprim or co-trimoxazole in the treatment of uncomplicated urinary tract infection in general practice. $\mathbf{J}$ Antimicrob Chemother. 1994;33(suppl_A):121-9.

12. Cagnacci S, Gualco L, Debbia E, Schito GC, Marchese A. European Emergence of CiprofloxacinResistant Escherichia coli Clonal Groups O25:H4-ST 131 and O15:K52:H1 Causing Community-Acquired Uncomplicated Cystitis. J Clin Microbiol. 2008;46(8):2605-12.

Cite this article as: Kaur R, Kumar V, Singh $\mathrm{H}$, Walia G, Sehgal A. Evaluation of efficacy and tolerability of nitrofurantoin versus ciprofloxacin in patients of urinary tract infection: a comparative study. Int J Basic Clin Pharmacol 2017;6:2690-4. 